\section{Flouroquinolones Resistant Escherichia Coli Isolated from Urine of Diabetics}

Sir,

Amongst the common risk factors of urinary tract infection (UTI) is immunosuppressed status, such as diabetes mellitus (DM), ${ }^{1}$ a metabolic disorder of prolonged high blood sugar levels. ${ }^{2}$ Hyperglycemia and glycosuria account for dysfunctional neutrophils. ${ }^{3}$

Patients with diabetes are predisposed to UTIs and the most frequently encountered microorganisms are Escherichia coli, Klebsiella pneumoniae, and Candida spp. 4

During May-October 2018, overall 1,520 urine specimens from diabetic inpatients were received in Rehman Medical Institute, Peshawar for culture and sensitivity testing, among which 486 (32\%) were catheterised patients. Seven hundred and ten specimens (46.7\%) yielded consideration bacteriuria; 223 urine samples $(14.6 \%)$ exhibited no growth and 587 urine specimens (38.6\%) displayed mixed growth. The different organisms isolated from urine specimen culture were $E$. coli, which was the most dominant, accounting for $57.7 \%(n=410)$ of the uropathogens. Others were: $15.2 \%$ Klebsiella pneumoniae $(n=108), 11.5 \%$ Pseudomonas aeruginosa $(n=82), 6.2 \%$ Enterococcus species $(n=44), 5.2 \%$ Proteus spp. $(\mathrm{n}=37), 2.7 \%$ Citrobacter spp. $(\mathrm{n}=19)$ and $1.4 \%$ Candida spp. $(\mathrm{n}=10)$.

Among total E. coli isolated, $56 \% \quad(n=230)$ were recovered from female inpatients and the rest $44 \%$ $(n=180)$ were from male admitted patients. Mean age of the patients was 58 years (age range on 40-75 years). One hundred and eighty four $(45 \%)$ isolates were recovered from patients of Cardiac Care Unit (CCU), while the remaining $55 \% \quad(n=226)$ were from patients admitted in different wards including Internal Medicine Ward $(n=127,31 \%)$, Urology Ward $(n=41,10 \%)$, Intensive Care Unit $(n=37,9 \%)$, and Surgical Ward $(n=21,5 \%)$. Primary susceptibility testing was performed in accordance with Clinical and Laboratory Standard Institute (CLSI) guidelines; 91\% $(n=373)$ were (norfloxacin and ciprofloxacin resistant and $9 \% \quad(n=37)$ were flouroquinolones sensitive. Among these, 85.2\% $\quad(n=350)$ isolates of $E$. coli were MDR.

During investigation, empirical therapy was perceived to have commenced in all cases. Among which, $(n=350)$ $82 \%$ received flouroquinolones, ceftriaxone $(n=37) 9 \%$, and in the remaining few amoxicillin-clavulanate, nitrofurantoin and trimethoprin-sulfamethoxazole were used.
In this study, $85.2 \%$ cultured E. coli were multi-drug resistant, which is relatively on rise when equated to others studies. Multidrug resistant E. coli was $52.9 \%$ in an Indian hospital setting 5 , and $7.1 \%$ in another research study by Sahm et al. in USA. 6 We perceived a substantial escalation in resistance to fluoroquinolones namely, norfloxacin and ciprofloxacin, that might be owing to empirical treatment practices for UTI. The same response was also noted in Uruguay, a state having widespread fluoroquinolones use for UTI empirical management. ${ }^{7}$ By this assessment, we have construed that fluoroquinolones will no longer be adequate for use against UTI due to $E$. coli in roughly next 20 years. The chief acumen for escalation in resistance is the lack of antibiotic stewardship measure, attributable to social and economic disputes in most nations. ${ }^{8}$ The escalating resistance to fluoroquinolones in $E$. coli from urinary tract infections may emerge from augmented prescription for UTI. E. coli resistance to these agents will probably upsurge further as fluoroquinolone use increases in future.

\section{CONFLICT OF INTEREST:}

Authors declared no conflict of interest.

\section{AUTHORS' CONTRIBUTION:}

MK: Conception or design of the work.

Al: Critical revision of the article.

FZ, TG: Data collection.

JA, FL: Data analysis and interpretation.

$\mathrm{SH}$ : Drafting the article.

\section{REFERENCES}

1. Dielubanza EJ, Schaeffer AJ. Urinary tract infections in women. Med Clin North Am 2011; 95:27-41.

2. Diabetes Fact Sheet $\mathrm{N}^{\circ} 312$ (2013): World Health Organization. http://www.who.int/mediacentre/factsheets/fs312/en/

3. Rizzi M, Trevisan R. Genitourinary infections in diabetic patients in the new era of diabetes therapy with sodiumglucose cotransporter-2 inhibitors. Nutr Metab Cardiovasc Dis 2016; 26:963-70.

4. Huang JJ, Tseng CC. Emphysematous pyelonephritis: Clinicoradiological classification, management, prognosis, and pathogenesis. Arch Intern Med 2000; 160:797-805.

5. Hasan AS, Nair D, Kaur J, Baweja G, Deb M, Aggarwal P. Resistance patterns of urinary isolates in a tertiary Indian hospital. J Ayub Med Coll Abbottabad 2007; 19:39-41.

6. Sahm DF, Thornsberry C, Mayfield DC, Jones ME, Karlowsky JA. Multidrug-resistant urinary tract isolates of Escherichia coli: Prevalence and patient demographics in the United States in 2000. Antimicrob Agents Chemother 2001; 45:1402-6.

7. Seija V, Frantchez V, Ventura V, Pintos M, González M. Factores asociados al desarrollo de infección urinaria de origen comunitario causada por Escherichia coli resistente a fluoroquinolonas. Rev Chilena Infectol 2014; 31:400-5.

8. Barriere SL. Clinical, economic and societal impact of antibiotic resistance. Expert Opin Pharmacother 2015; 16:151-3. 
Maria Khan', Aamer Ikram², Fatima Zahra ${ }^{3}$, Jawad Ahmed', Tahir Ghafoor ${ }^{3}$, Farida Lalani ${ }^{3}$, Shahzad Hassan ${ }^{4}$

${ }^{1}$ Department of Microbiology, Rehman Medical Institute, Peshawar, Pakistan

2 National Institute of Health, Islamabad, Pakistan

3 Department of Microbiology, Armed Forces Institute of Pathology, Rawalpindi, Pakistan
4 Khyber Medical University, Peshawar Pakistan

Correspondence to: Dr. Maria Khan, Department of Pathology, Rehman Medical Institute, Peshawar Pakistan

E-mail:kmaria22@hotmail.com

Received: October 15, 2018; Revised: April 8, 2019; Accepted: April 12, 2019

.......... 